\title{
Effects of Two Mutations Detected in Medium Chain Acyl-CoA Dehydrogenase (MCAD)-deficient Patients on Folding, Oligomer Assembly, and Stability of MCAD Enzyme*
}

\author{
Peter Bross $\ddagger \S$, Charlotte Jespersen $\ddagger$, Thomas G. Jensen $\rceil$, Brage S. Andresen $\ddagger$, \\ Morten Juhl Kristensen $\ddagger$, Vibeke Winter $\ddagger$, Andreas Nandy\|, Franz Kräutle\|, \\ Sandro Ghisla $\|$, Lars Bolund 1 , Jung-Ja P. Kim**, and Niels Gregersen $\neq$ \\ From the $\ddagger$ Center for Medical Molecular Biology, Aarhus University Hospital and Faculty of Health Sciences, \\ Skejby Sygehus, 8200 Aarhus N, Denmark, IInstitute for Human Genetics, Aarhus University, 8000 Aarhus C, \\ Denmark, **Department of Biochemistry, Medical College of Wisconsin, Milwaukee, Wisconsin 53226, and \\ |Faculty for Biology, University of Konstanz, 7750 Konstanz, Federal Republic of Germany
}

We have used expression of human medium chain acyl-CoA dehydrogenase (MCAD) in Escherichia coli as a model system for dissecting the molecular effects of two mutations detected in patients with MCAD deficiency. We demonstrate that the R28C mutation predominantly affects polypeptide folding. The amounts of active R28C mutant enzyme produced could be modulated between undetectable to $100 \%$ of the wild-type control by manipulating the level of available chaperonins and the growth temperature. For the prevalent K304E mutation, however, the amounts of active mutant enzyme could be modulated only in a range from undetectable to approximately $50 \%$ of the wild-type, and the assembled mutant enzyme displayed a decreased thermal stability. Two artificially constructed mutants (K304Q and K304E/D346K) yielded clearly higher amounts of active MCAD enzyme than the $\mathrm{K304E}$ mutant but were also responsive to chaperonin co-overexpression and growth at low temperature. The thermal stability profile of the K304E/D346K double mutant was shifted to even lower temperatures than that of the K304E mutant, whereas that of the K304Q mutant was closely similar to the wild-type. Taken together, the results show that the K304E mutation affects (i) polypeptide folding due to elimination of the positively charged lysine and (ii) oligomer assembly and stability due to replacement of lysine 304 with the negatively charged glutamic acid.

Medium chain acyl-CoA dehydrogenase $\left(\mathrm{MCAD}^{1} ; \mathrm{EC}\right.$ 1.3.99.3) is one of four related enzymes with different chain length specificity that catalyze the initial step in mitochondrial $\beta$-oxidation of straight chain fatty acids $(1,2)$. The active enzyme consists of four identical subunits, each containing one molecule of FAD. MCAD has received particular interest, since MCAD deficiency is by far the most frequently detected inher-

* This work was supported by the Danish Medical Research Council and the Danish Center for Human Genome Research. The costs of publication of this article were defrayed in part by the payment of page charges. This article must therefore be hereby marked "advertisement" in accordance with 18 U.S.C. Section 1734 solely to indicate this fact.

$\S$ To whom correspondence should be addressed: Center for Medical Molecular Biology, Aarhus University Hospital, Skejby Sygehus, Brendstrupgårdsvej, 8200 Århus N, Denmark. Tel.: 45-89-495150; Fax: 45-89-496018; E-mail: bross@biobase.dk.

${ }^{1}$ The abbreviations used are: MCAD, medium chain acyl-CoA dehy. drogenase; hsp60, heat-shock protein 60; hsp70, heat-shock protein 70 PAGE, polyacrylamide gel electrophoresis; PCR, polymerase chain reaction. itable defect in mitochondrial $\beta$-oxidation (3). Disease manifestation in clinically affected patients presents with life-threatening attacks induced by fasting stress, often in connection with viral infections (4). Patients present with clinical symptoms typically at the age of 1-4 years, and approximately $20 \%$ of the patients detected so far died during the first attack (4). When fasting is avoided, patients are usually asymptomatic.

Besides a series of rare point mutations, short insertions, and deletions (5-7), one prevalent point mutation (8-11) is present in more than $90 \%$ of the alleles in patients with MCAD deficiency (5). This mutation is an $\mathrm{A} \rightarrow \mathrm{G}$ transition at position 985 of the cDNA sequence (G985), leading to replacement of lysine 304 in the mature protein with glutamic acid (K304E). The calculated homozygote frequency of the G985 mutation in Northwest European and North American Caucasians is in the range of $1 / 6,400$ to $1 / 40,000$ (12). This frequency is much higher than the actual number of clinically detected symptomatic cases, suggesting that many homozygotes are clinically asymptomatic $(12,13)$. The reason for the phenotypic variation from sudden unexpected death in childhood to asymptomatic presentation (4) is not clear

To delineate the pathological mechanism, the molecular consequences of the K304E mutation have been investigated. We have used an approach where mature human MCAD was expressed in Escherichia coli with and without co-overexpression of the chaperonins GroEL and GroES, two proteins that assist in folding of newly synthesized polypeptide chains (for a recent review, see Ref. 14). We demonstrated that K304E mutant MCAD can be partially rescued from misfolding in the presence of excess GroESL and that a minor portion of the expressed K304E MCAD protein folded into the native tetrameric structure with a specific activity in the range of the wild-type enzyme (15). From these data and comparative analysis of a MCAD variant with the artificially constructed K304Q mutation, we proposed that the K304E mutation has a 2-fold effect: one on polypeptide folding, due to elimination of the positive charge in the side chain, and another one on oligomer assembly, due to the introduction of a negatively charged side chain. The effect on oligomer assembly is supported by data from the crystal structure of porcine MCAD (16), which show that lysine 304 is situated in helix $H$, forming part of the subunit interface. With in vitro translation experiments in the presence of rat liver mitochondria, Saijo et al. (17) showed that, after import into mitochondria, MCAD chains transiently interact with the mitochondrial heat shock protein 70 (hsp70) and subsequently with chaperonin hsp60, a homolog of the bacterial GroEL, before assembled MCAD tetramers can be observed. The se- 
quence of events was similar for K304E mutant MCAD, but this protein remained associated with hsp60 for longer time periods, indicating that folding and/or oligomer assembly is impaired. These authors could not discriminate between an effect of the mutation on monomer folding or oligomer assembly.

Another missense mutation in the MCAD gene (T157), which has been detected in two patients in compound heterozygosity with the G985 mutation, results in the substitution of arginine 28 with cysteine (R28C) (6). One of the patients died unexpectedly at day 3 after birth, while the other had clinical symptoms at 8 months of age (18), indicating the potential severity and variable expression of the mutation. In COS-7 cell-based expression experiments the R28C mutant enzyme displayed only moderately decreased MCAD activity levels (50-100\% of wildtype) $(6,19)$. Expression of this mutant protein in $E$. coli revealed a strong effect of high chaperonin levels on the produced amounts of active R28C mutant MCAD (19).

Studies with mutants in the coat and tailspike proteins of phage P22 have identified a group of so-called temperaturesensitive folding mutations which primarily affect the yield of correctly folded polypeptide, but not, or only to a minor extent, the stability and functional integrity of the native structure once it is formed $(20,21)$. It has been demonstrated that cooverexpression of the GroESL chaperonins could suppress the folding defects of temperature-sensitive folding mutants in the phage P22 coat protein but not in the tailspike protein (22).

The aim of the present study was to distinguish the effect of the K304E and R28C mutations on MCAD polypeptide folding from effects on oligomer assembly and stability of the active tetramer in order to explore to what extent the cellular folding machinery may be able to modulate manifestation of the defect in patients harboring these mutations. Folding conditions were optimized by growth at low temperature and co-overexpression of the GroESL chaperonins, and the amounts of active mutant MCAD produced as well as the temperature stability of the tetrameric enzyme variants were analyzed. The effect of the charge reversal in the prevalent $\mathrm{K} 304 \mathrm{E}$ mutation was investigated further by introducing second site mutations in charged residues that reestablish the net charge constellation around residue 304 .

\section{EXPERIMENTAL PROCEDURES}

Plasmids-The previously used plasmid pBMCK2- ${ }^{-}$(8), which carries a gene encoding the mature part of human MCAD preceded by an artificial initiator methionine under control of the lac promoter, was modified in order to allow straightforward subcloning of PCR amplified MCAD cDNA derived from patient material. For this purpose $\mathrm{pB}$ MCK2 $^{-}$was modified in a series of steps involving standard PCR and cloning techniques in the following way. A HindIII site included in the PCR primer 1327-HindIII (5'-TTTTCTGAAGCTTAAACAGTGGCTTGTGTTC- $3^{\prime}$ ) (7) was introduced at the $3^{\prime}$ end of the cDNA insert; the linker fragment of the parent vector (pBluescriptKS ${ }^{-}$; Stratagene) between the HindIII and BamHI sites was reintroduced followed by deletion of the sites between $E c o$ RV and SmaI by cleavage with these enzymes and religation. The final product was designated $\mathrm{pWt}$. The $\mathrm{K} 304 \mathrm{E}, \mathrm{R} 28 \mathrm{C}$, and $\mathrm{K} 304 \mathrm{Q}$ mutations were introduced into $\mathrm{pWt}$ by subcloning of appropriate fragments from plasmids described previously $(6,15)$ producing a set of plasmids which only differ in their respective mutation sites.

For construction of the second site mutations, plasmids pWTMCAD-2 encoding wild-type human MCAD protein and p985 encoding $\mathrm{K} 304 \mathrm{E}$ mutant protein, described previously $(15,23)$, were cleaved with Pst I and HindII or PstI and BamHI respectively, and the fragments comprising the MCAD cDNA insert were purified by gel electrophoresis. These fragments were then used as templates for a megaprimer PCR mutagenesis procedure described by Kuipers et $a l$. (24) with the following modifications. The megaprimer was amplified using primer 1327 HindIII and either one of the mutagenic primers pri- $(s)+959\left(5^{\prime}\right.$-CATTTATGCTGGCTAAAATGG-3'; E300K mutation) or pri-(s)+1099 $\left(5^{\prime}\right.$ CAGTTAGCTACTAAAGCTGTGCAG-3'; D346K mutation). After 20 cycles of PCR, the amplified fragments were purified by ultrafiltration
(Centricon 100; Amicon). A second round of PCR was performed with each of the megaprimers, a new universal primer pri- $(s)+378\left(5^{\prime}-\right.$ TTCTTTGGGGCAAATGCCTA-3') and the same templates as in the first PCR. The PCR product was precipitated, cleaved at the internal $E c o R I$ site and the introduced HindIII, site and ligated into $\mathrm{pWt}$ cleaved with the same enzymes. The ligation product was transformed into $E$. coli JM109. Clones were checked for the absence of PCR errors.

Growth of Bacterial Cells and Analysis of Extracts-JM109 cells transformed with the respective MCAD plasmid and $\mathrm{pGroESL}$ encoding the GroESL (25) proteins or the control plasmid $\mathrm{pCaP}$ (15) were grown at $28,31,34,37$, or $40^{\circ} \mathrm{C}$ in a shaking water bath until an $\mathrm{OD}_{532}$ of $0.7-1.0$ was reached. The cells were then induced with $1 \mathrm{~mm}$ isopropyl$\beta$-D-thiogalactopyranoside for $3 \mathrm{~h}$ and harvested. Disruption of cells, SDS-PAGE, native PAGE, and Western blotting were performed as described elsewhere (15). MCAD enzyme activity in bacterial lysates was determined with the ferricenium ion-based colorimetric assay as described by Lehman et al. (26).

Thermal Stability Profiles-For determination of the thermal stability profiles, aliquots of $12-22 \mu \mathrm{l}$ of extracts from cells expressing the respective MCAD variant and the GroESL chaperonins were incubated at various temperatures for $10 \mathrm{~min}$ and chilled on ice. MCAD enzyme activity was subsequently measured with the colorimetric ferricenium ion assay as above.

Cultivation of Lymphoblastoid Cells and Two-dimensional Gel Electrophoresis-Epstein-Barr virus-transformed lymphoblastoid cells were cultivated at various temperatures for 14 days using RPMI 1640 medium supplemented with $10 \%$ fetal calf serum. Preparation of samples and two-dimensional gel electrophoresis followed by Western blotting were carried out as previously described (27). MCAD enzyme activity in extracts from lymphoblastoid cells was measured with the mass spectrometric product formation assay (28).

\section{RESULTS}

Influence of Temperature and Amount of Chaperonins on Solubility and Yield of Active Wild-type and Mutant MCAD Variants-The mature part of wild-type MCAD and the R28C, $\mathrm{K} 304 \mathrm{E}$, and $\mathrm{K} 304 \mathrm{Q}$ mutant variants were expressed in $E$. coli with and without co-overexpression of the GroESL chaperonins. Cells were cultured at a range of temperatures between 28 and $40^{\circ} \mathrm{C}$.

For the wild-type protein, the amounts of active enzyme produced in the samples, both with and without GroESL overexpression, is similar at 28,31 , and $34^{\circ} \mathrm{C}$ (Fig. 1). There is only slightly more active enzyme present at high chaperonin levels. This is also reflected by a low amount of insoluble MCAD material in the samples without GroESL co-overexpression. At $37^{\circ} \mathrm{C}$, the difference between the samples with and without GroESL co-overexpression is somewhat higher, and at $40^{\circ} \mathrm{C}$ GroESL co-overexpression increases the yield of active MCAD by a factor of approximately 2 , indicating that the amount of GroESL becomes rate-limiting for the wild-type protein at these temperatures. Concomitantly, the amounts of insoluble wild-type MCAD protein are increased at these temperatures, and an effect of GroESL co-overexpression on the partition between soluble and insoluble wild-type MCAD becomes evident.

For all three mutant proteins investigated, GroESL co-overexpression has a strong positive effect on the amounts of active mutant enzyme produced. The chaperonin effect is most dramatic at $34^{\circ} \mathrm{C}$ and becomes smaller at lower temperatures. An exception is the K304Q mutant which displays a strong chaperonin effect at 34,37 , and $40^{\circ} \mathrm{C}$. With GroESL co-overexpression, R28C and K304Q MCAD reach relative activity levels in the range of the wild-type enzyme at $34^{\circ} \mathrm{C}$. The activity level of the K304E mutant MCAD with GroESL co-overexpression increases to approximately half of the wild-type activity at $34^{\circ} \mathrm{C}$ and remains at this level at 31 and $28^{\circ} \mathrm{C}$. This suggests that this is a limit which cannot be surpassed by further improving the folding conditions.

All mutant variants display a high proportion of insoluble MCAD protein at 37 and $40^{\circ} \mathrm{C}$. The total amount of MCAD mutant material detected at these temperatures is similar to 


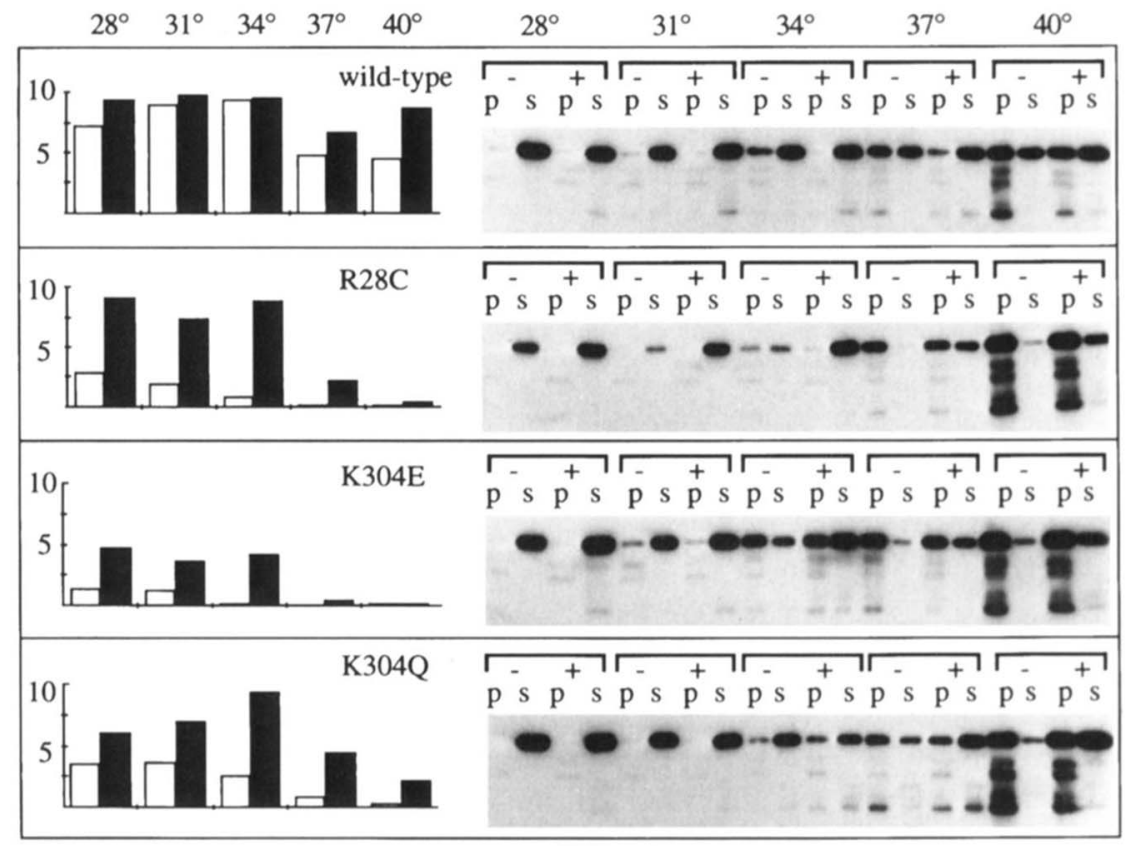

FIG. 1. Solubility and relative MCAD enzyme activity of MCAD variants expressed at different culture temperatures in $E$. coli with or without co-overexpression of the GroESL chaperonins. $E$. coli JM109 cells transformed with plasmids containing the cDNA sequence for the mature part of wild-type, R28C, K304E, or K304Q MCAD were grown and induced for $3 \mathrm{~h}$ at the temperature indicated. Cells were harvested, disrupted, and split into soluble $(s)$ and insoluble $(p)$ fractions as described under "Experimental Procedures." Left panel, MCAD enzyme activity in the soluble fraction was measured with the colorimetric assay using the ferricenium ion as electron acceptor. The scale is in micromoles of ferricenium reduced per h per mg of total soluble protein. Columns represent the average from two measurements (open columns, without GroESL co-overexpression; closed columns, with GroESL co-overexpression). Right panel, aliquots containing $1.25 \mu \mathrm{g}$ of total protein of the soluble fraction and a corresponding aliquot of the solubilized pellet fraction were subjected to SDS-PAGE followed by Western blotting with rabbit antiporcine MCAD antibodies. (+) with co-overexpression of the GroESL genes, $(-)$ without co-overexpression of the GroESL genes.

the amount of wild-type protein, indicating that transcription and translation rates are identical for all variants. At low temperatures and without GroESL co-overexpression, the amount of mutant MCAD material detected in the pellet fraction is low. However, in some of these samples (e.g. R28C at 34 and $31{ }^{\circ} \mathrm{C}$ ) only slightly higher amounts are observed in a soluble form. The decreased amount of insoluble MCAD material in these samples is therefore likely to be due both to conversion of a larger proportion of the polypeptide chains into soluble species and to increased degradation of accumulating MCAD folding intermediates.

Thermal Stability of the Mutant Enzymes-In order to investigate whether the mutations affect the stability of the assembled enzyme, we determined the thermal inactivation profiles of the mutant MCAD enzymes (Fig. 2). K304E MCAD shows a temperature stability profile clearly shifted to lower temperatures, indicating that this mutation has an effect on the stability of the tetramer once it is formed. Almost identical curves could be observed for wild-type, R28C, and K304Q MCAD with the curve for the K304Q mutant being marginally but consistently steeper. This indicates that folded and assembled R28C MCAD is as stable, and the K304Q mutant only slightly less stable than the wild-type enzyme.

Temperature-sensitive Yield of K304E Mutant MCAD in Patient Cells-To test the relevance of the results obtained in the $E$. coli model system, we analyzed the steady state amount of K304E MCAD in lymphoblastoid cells as a function of the temperature at which the cells were cultivated. MCAD enzyme activity was measured in lymphoblastoid cells cultivated at 34 , 37 , or $39^{\circ} \mathrm{C}$, respectively. Cells homozygous or heterozygous for the K304E mutation and normal controls were used. Table I shows that the activity values for the wild-type control cells are constant between 34 and $37^{\circ} \mathrm{C}$ and decreased to less than half at $39{ }^{\circ} \mathrm{C}$. A similar picture is observed for the cells heterozygous for the K304E mutation. The respective activity levels are about half of those for the wild-type control, indicating that the contribution of the mutant enzyme to the activity level is insignificant. The cells homozygous for the K304E mutation display very low levels at 39 and $37{ }^{\circ} \mathrm{C}$ and an approximately 3 -fold increased relative activity at $34{ }^{\circ} \mathrm{C}$.

This result was further confirmed by a qualitative determination of the ratio between wild-type and K304E MCAD material in the cells heterozygous for the K304E mutation. We have previously shown that wild-type and K304E mutant MCAD can be separated by two-dimensional gel electrophoresis. Two spots each, one modified and one nonmodified form, could be observed for wild-type and K304E MCAD, respectively (27). The results are depicted in Fig. 3. Clearly, the steadystate amount of K304E MCAD relative to the wild-type protein is higher in the cells cultivated at $34^{\circ} \mathrm{C}$ than at 37 or $39^{\circ} \mathrm{C}$. This demonstrates that the relative amount of K304E MCAD protein is temperature-sensitive in these cells.

Analysis of Interactions of Lysine 304 with Other Charged Residues in the Native Structure-If the effect of the K304E mutation on polypeptide folding is distinct from those on oligomer assembly and stability, it should be possible to compensate for this effect separately by reconstituting the charge interaction which is distorted in the K304E mutant. The crystal structure of porcine MCAD is available at $2.4 \AA$ resolution (16). Fig. $4 A$ gives an enlarged view of the vicinity of lysine 304 , showing distances between relevant atoms. There are two negatively charged groups, aspartic acid 300 and aspartic acid 346 in the vicinity of the amino group of lysine 304. Aspartic acid 300 forms a salt bridge with arginine 383 in the neighboring subunit. Lysine 304 forms a hydrogen bond with the $\gamma$-carbonyl oxygen of glutamine 342. A similar hydrogen bond can also be formed with the $\gamma$-amido- $\mathrm{NH}_{2}$ group of glutamine 342 , if lysine 304 is replaced by glutamic acid (16). The distances between the positively charged group of lysine 304 and the other charged atoms are greater than expected for genuine salt 


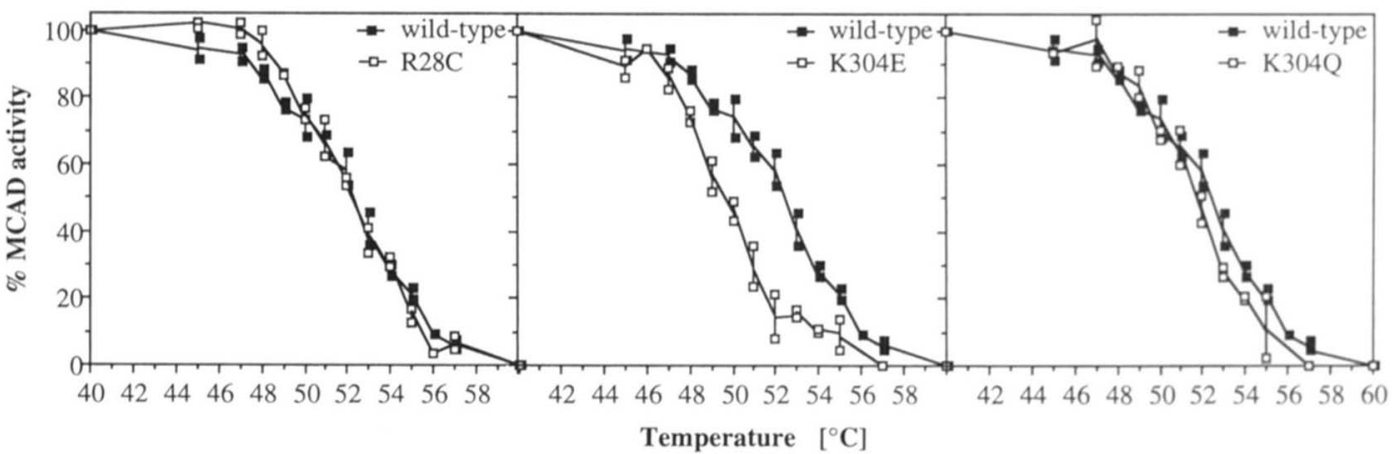

FIG. 2. Thermal inactivation profiles of mutant MCAD enzymes. Aliquots from the soluble fractions of cells expressing wild-type, R28C $\mathrm{K} 304 \mathrm{E}$, or K304Q MCAD were incubated at various temperatures for $10 \mathrm{~min}$, and MCAD enzyme activity was measured afterward. Residual enzyme activity (percentage of the activity measured at $40{ }^{\circ} \mathrm{C}$ ) is plotted versus the incubation temperature. The values from two independent experiments each with extracts from induction experiments performed at 28 or $31^{\circ} \mathrm{C}$ are shown. The total protein concentrations in the extracts were in the range of $2.6-2.9 \mathrm{mg} / \mathrm{ml}\left(28^{\circ} \mathrm{C}\right)$ and $2.1-2.5 \mathrm{mg} / \mathrm{ml}\left(31^{\circ} \mathrm{C}\right)$. The profile obtained with an extract from cells expressing wild-type MCAD is shown for comparison.

TABLE I

MCAD enzyme activity in extracts from lymphoblastoid cells cultivated at 34,37 , or $39^{\circ} \mathrm{C}$. Cells (normal controls, cells heterozygous for the K304E mutation and cells from a patient homozygous for the K304E mutation) were disrupted by sonication and centrifuged, and MCAD enzyme activity was measured with the mass spectrometric product formation assay (26). Both values from measurements in duplicates are given in nanomoles of $\mathrm{OH}$-octanoic acid formed per $\mathrm{h}$ per $\mathrm{mg}$ of soluble protein.

\begin{tabular}{lccc}
\hline $\begin{array}{c}\text { MCAD } \\
\text { genotype }^{a}\end{array}$ & $34{ }^{\circ} \mathrm{C}$ & $37{ }^{\circ} \mathrm{C}$ & $39{ }^{\circ} \mathrm{C}$ \\
\hline wt/wt & $185 / 222$ & $115 / 294$ & $70 / 67$ \\
wt/K304E & $94 / 65$ & $87 / 76$ & $32 / 24$ \\
$\mathrm{~K} 304 \mathrm{E} / \mathrm{K} 304 \mathrm{E}$ & $19 / 17$ & $7 / 7$ & $3 / 3$ \\
\hline
\end{tabular}

${ }^{a}$ wt, wild-type.

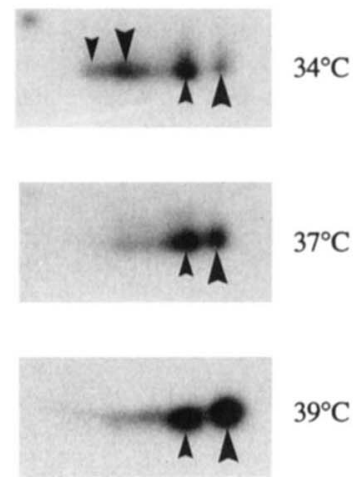

FIG. 3. Two-dimensional gel electrophoresis of lymphoblastoid cells cultivated at different temperatures. Lymphoblastoid cells carrying the K304E mutation on one and wild-type MCAD on the other allele were grown at 34,37 , or $39^{\circ} \mathrm{C}$, respectively, harvested, and solubilized in lysis buffer. Aliquots corresponding to $127 \mu \mathrm{g}\left(34^{\circ} \mathrm{C}\right), 111$ $\mu \mathrm{g}\left(37^{\circ} \mathrm{C}\right)$, and $183 \mu \mathrm{g}\left(39^{\circ} \mathrm{C}\right)$ total protein were subjected to twodimensional gel electrophoresis followed by Western blotting with rabbit antiporcine MCAD antibodies as described elsewhere (27). Sections of the gels are shown and the spots corresponding to unmodified (large arrowhead) and modified (small arrowhead) wild-type (upward arrow. heads) and K304E mutant (downward arrowheads) MCAD are marked

bridge interactions. All possible interactions of lysine 304 with other charged side chains are within the monomer, and there is no indication that lysine 304 directly interacts with residues of the neighboring subunit. Preliminary results with the human enzyme demonstrate that the structure is highly similar to that of porcine $\mathrm{MCAD} .^{2}$ Comparison of the conservation of these residues in the sequences of other acyl-CoA dehydrogenases (Fig. $4 B$ ) reveals that the negative charge of residue 300 as well

${ }^{2}$ Jung-Ja P. Kim, unpublished results. as arginine 383 are conserved in all enzymes. A negative charge at position 346 is present in all cases where there is a positively charged residue at position 304 . It may therefore be speculated that a charge repulsion between glutamic acid 304 and glutamic acid 300 and/or aspartic acid 346 in the human K304E MCAD mutant could propagate a structural distortion within the monomer.

To determine whether such charge interactions are relevant for the effect of the K304E mutation on oligomer assembly and stability, we constructed the second site mutants K304E/ D346K and E300K/K304E, thereby reconstituting opposite charge constellations in the vicinity of the carboxyl group of glutamic acid 304. The single mutants D346K and E300K were also constructed in order to analyze the effect of the mutations as such.

The mutants were expressed at 28 and $37^{\circ} \mathrm{C}$ in $E$. coli with and without co-overexpression of the GroESL chaperonins. As shown in Fig. $5 A$, the E300K single and the E300K/K304E double mutant variants yield very low or undetectable amounts of MCAD activity when produced at $37^{\circ} \mathrm{C}$, although considerable amounts of soluble $\mathrm{MCAD}$ protein were observed in the samples with GroESL co-overexpression (data not shown). When the cells were grown at $28^{\circ} \mathrm{C}$, increased amounts of active $\mathrm{E} 300 \mathrm{~K}$ single and $\mathrm{E} 300 \mathrm{~K} / \mathrm{K} 304 \mathrm{E}$ double mutant MCAD could be observed, with the double mutant displaying lower levels than the single mutant. Analysis of these extracts by native PAGE/Western blotting revealed an amount of tetramer roughly corresponding to the MCAD activity measured (data not shown). This means that the effect of the E300K mutation increases rather than compensates the effects of the K304E mutation. The E300K and E300K/K304E mutant enzymes were very unstable as indicated by a dramatically decreased MCAD enzyme activity level after only one freeze/thaw cycle of the extracts (data not shown).

Strikingly, the amount of active K304E/D346K double mutant protein is clearly increased in comparison to both the K304E and the D346K single mutants. The effect is most remarkable in the $37^{\circ} \mathrm{C}$ series. The amounts of active $\mathrm{K} 304 \mathrm{E} /$ D346K MCAD produced are strongly responsive to chaperonin co-overexpression, indicating that the effect on folding is not overcome. The characteristics of the K304E/D346K double mutant are similar to those of the K304Q mutant (see Fig. 2).

The thermal stability profile of the K304E/D346K double mutant was determined and is displayed in Fig. $5 B$. The profiles for wild-type and K304E MCAD are replotted from Fig. 2 for comparison. The profile for the K304E/D346K double mutant is shifted to even lower temperatures than the K304E single mutant. We also attempted to determine the profile for 
FIG. 4. A, an enlarged view of the vicinity of lysine 304 of a monomer of porcine MCAD. Helices $\mathrm{H}$ and $\mathrm{I}$ are shown in ribbons and side chain atoms of lysine 304, aspartic acid 346 , glutamine 342 , aspartic acid 300 , and the main chain carbonyl atoms of glutamine 342 are shown in solid balls. The side chain of arginine 383 of the neighboring monomer is represented by open ball-and-stick. Distances between polar atoms in ångstroms are shown with dotted lines. The figure was drawn using the program Molscript (29). $B$, conservation of residues $300,304,342,346$, and 383 (MCAD numbering) in the acyl-CoA dehydrogenase gene family. Nomenclature (cDNA sequence reference in parentheses): $L C A D$, long chain acyl-CoA dehydrogenase (30, 31); MCAD (32, 33); $S C A D$, short chain acyl-CoA dehydrogenase $(30,34), I V D$, isovaleryl-CoA dehydrogenase $(30,35)$; small letters refer to the species ( $h$, human; $r$, rat; $p$, porcine).

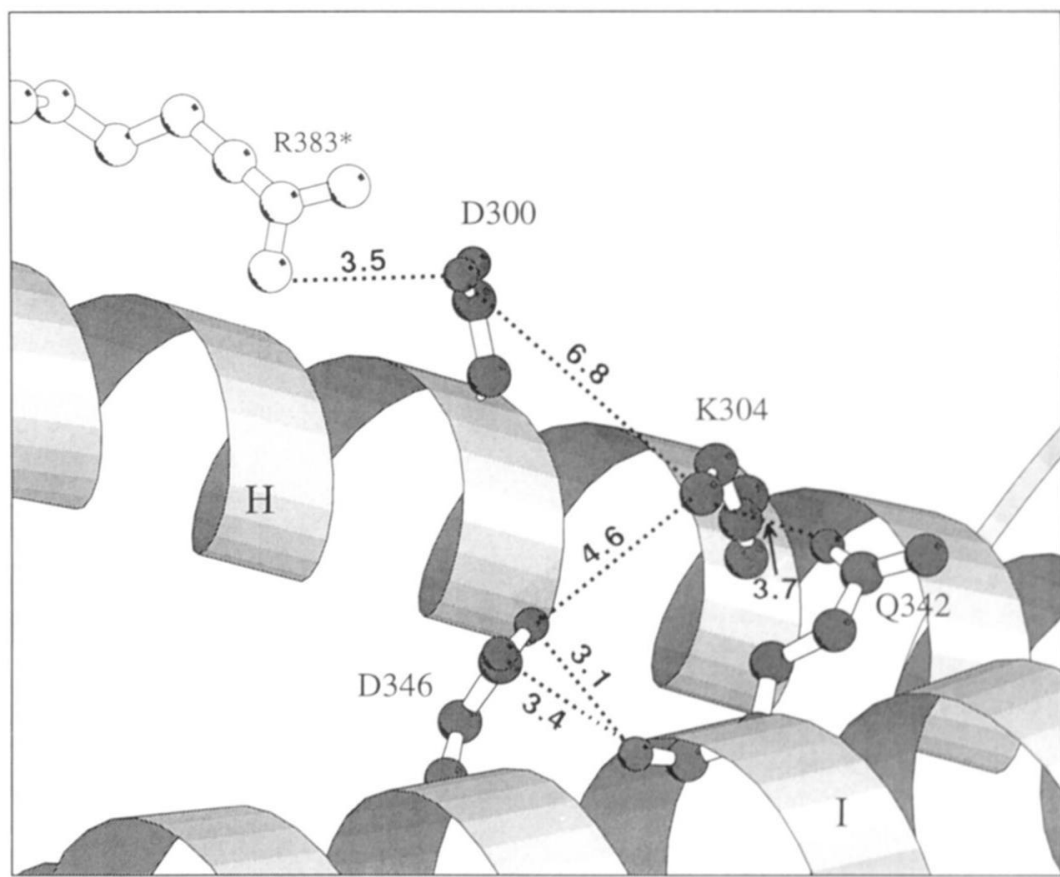

B

$\begin{array}{lccccc} & 300 & 304 & 342 & 346 & 383 \\ \text { hMCAD } & \text { E } & \mathrm{K} & \mathrm{Q} & \mathrm{D} & \mathrm{R} \\ \text { pMCAD } & \mathrm{D} & \mathrm{K} & \mathrm{Q} & \mathrm{D} & \mathrm{R} \\ \text { rMCAD } & \mathrm{E} & \mathrm{K} & \mathrm{Q} & \mathrm{D} & \mathrm{R} \\ \text { hSCAD } & \mathrm{D} & \mathrm{A} & \mathrm{A} & \mathrm{Q} & \mathrm{R} \\ \text { rSCAD } & \mathrm{D} & \mathrm{A} & \mathrm{A} & \mathrm{Q} & \mathrm{R} \\ \text { hLCAD } & \mathrm{E} & \mathrm{H} & \mathrm{S} & \mathrm{D} & \mathrm{K} \\ \text { rLCAD } & \mathrm{E} & \mathrm{N} & \mathrm{T} & \mathrm{Q} & \mathrm{K} \\ \text { hIVD } & \mathrm{D} & \mathrm{R} & \mathrm{Q} & \mathrm{D} & \mathrm{R} \\ \text { rIVD } & \mathrm{D} & \mathrm{R} & \mathrm{Q} & \mathrm{D} & \mathrm{R}\end{array}$

the D346K single mutant. It turned out that this mutant protein was very unstable in the handling. While it was not possible to obtain a reliable profile, it was apparent that the D346K enzyme was inactivated at temperatures above $30{ }^{\circ} \mathrm{C}$. This shows that the D346K mutation itself strongly compromises enzyme stability, and that this effect is to some extent compensated by the K304E mutation in the K304E/D346K double mutant.

\section{DISCUSSION}

In the present study we have exploited both chaperonin co-overexpression and growth at low temperatures in an $E$. coli model system to maximize the folding yield and thereby overcome the effect of mutations in MCAD which impair folding of the polypeptide. The percentage of wild-type level to which the mutant MCAD variants could be restored by these treatments was assessed, and this was used to evaluate to what extent the folding environment can modulate the steady state level of active MCAD of two disease-causing mutant variants.

Our experiments demonstrate that the R28C mutation primarily affects polypeptide folding rather than the stability of the assembled enzyme. This mutation may thus be compared to the group of temperature-sensitive folding mutations investigated in detail for the phage P22 tailspike and coat proteins, which are impaired in kinetics of folding but not in the stability of the folded state. Manipulation of the growth temperature and the availability of chaperonins in the $E$. coli model system made it possible to modulate the relative amounts of active
R28C MCAD from undetectable to levels in the range of the wild-type. In a parallel study we had shown that expression of the R28C mutant in eucaryotic COS-7 cells produced 50-100\% of the amounts observed for wild-type $\operatorname{MCAD}(6,19)$, indicating that a particularly high capacity of the folding machinery in COS-7 cells may camouflage the folding problem. This mutant protein may thus be expected to be strongly sensitive to factors affecting the folding capacity of the cell. Arginine 28 is situated in helix A and forms a salt bridge with glutamic acid 86 in the neighboring helix D. Both arginine 28 and glutamic acid 86 are exposed on the surface of the tetramer (6). This salt bridge does apparently not contribute significantly to the thermal stability of the native tetramer. It has been observed for other proteins that salt bridges located at the surface of the molecule often are not critical for stability (36). It may be noted here that a mutation (R22W) at the homologous position in short chain acyl-CoA dehydrogenase has been detected in a patient with short chain acyl-CoA dehydrogenase deficiency (37), suggesting a similar molecular mechanism also in this case.

For the K304E mutant variant optimization of the folding conditions by lowering the growth temperature and co-overexpressing the GroESL chaperonins resulted in a maximum level of approximately $40-50 \%$ of the wild-type. This yield was obtained at 34,31 , and $28{ }^{\circ} \mathrm{C}$, indicating that a further improvement of the folding conditions had no further effect. The observed decreased thermal stability indicated that the mutation does not confer a classical temperature-sensitive folding phe- 
A

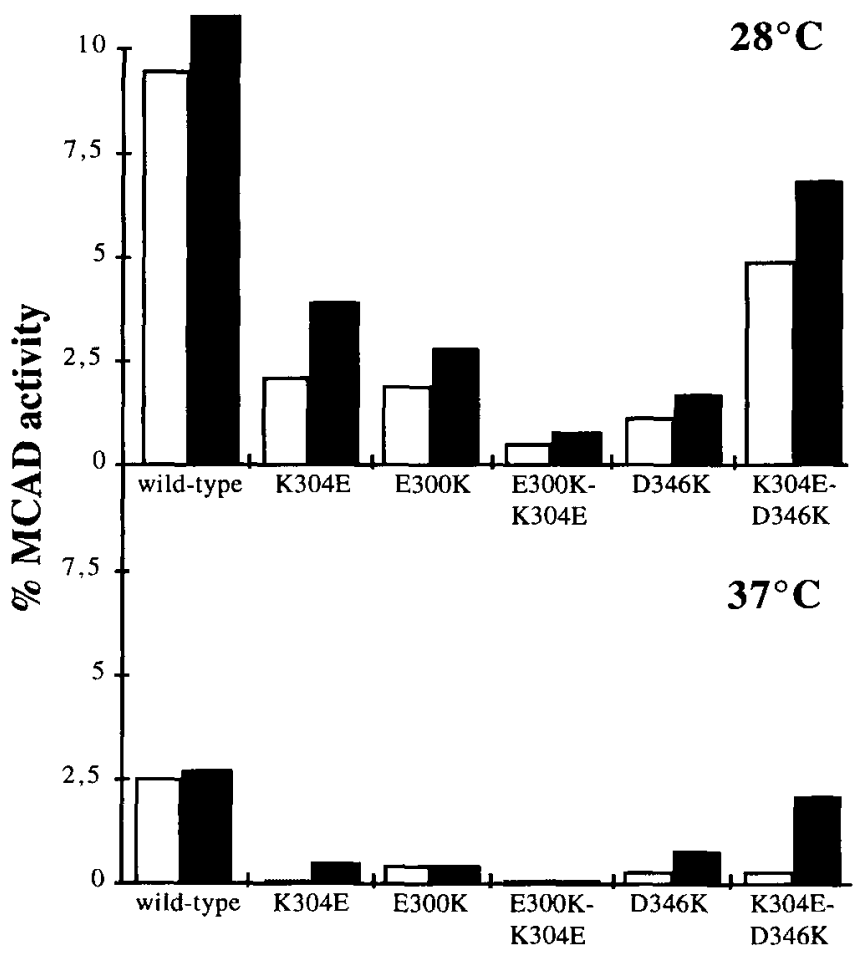

B

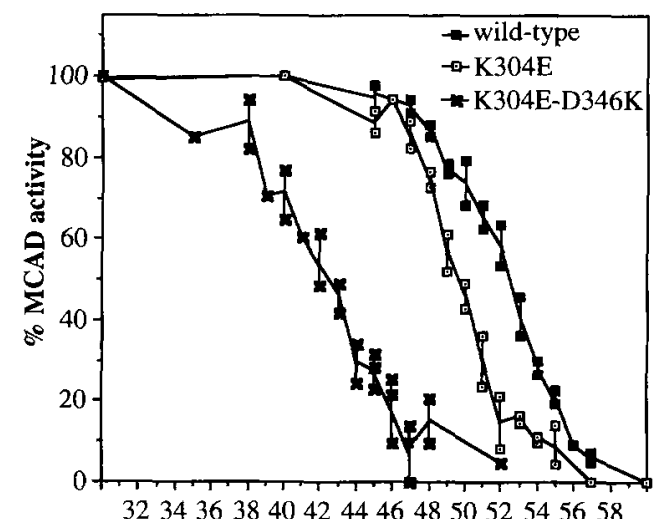

Temperature $\left[{ }^{\circ} \mathrm{C}\right]$

FIG. 5. A, enzyme activity levels of MCAD variants with second site mutations. E. coli JM109 cells transformed with a plasmid encoding the mature part of the respective MCAD mutant variants as indicated and either the plasmid encoding the GroESL (closed columns) genes or the control plasmid (open columns) were grown and induced at 28 or $37^{\circ} \mathrm{C}$ respectively. Cells were harvested, disrupted, and split into soluble and insoluble fractions as described under "Experimental Procedures." MCAD enzyme activity in the soluble fractions was measured with the colorimetric assay using the ferricenium ion as electron acceptor. The scale is in micromoles of ferricenium reduced per h per mg of total soluble protein. Columns represent the average from two measurements. $B$, thermal inactivation profile of the K304E/D346K double mutant. Aliquots from extracts expressing K304E/D346K mutant $M C A D$ were incubated at various temperatures for $10 \mathrm{~min}$, and MCAD enzyme activity was measured subsequently. Residual enzyme activity expressed as the percentage of the activity measured after $10 \mathrm{~min}$ of incubation at $30^{\circ} \mathrm{C}$ is plotted versus the incubation temperature. The profiles for wild-type and K304E MCAD determined in the same way (see Fig. 2) are shown for comparison.

notype, but rather impairs both polypeptide folding and the thermal stability of the folded and assembled mutant tetramer.

The data obtained previously with the K304E and the artificially constructed mutant K304Q suggested that replacement of lysine 304 with either glutamic acid or glutamine resulted in impaired folding kinetics. Based on the discrepancy observed between the properties of the $\mathrm{K} 304 \mathrm{Q}$ and the $\mathrm{K} 304 \mathrm{E}$ mutant, we proposed (15) that charge reversal in the $\mathrm{K} 304 \mathrm{E}$ variant may induce a structural distortion in the monomer that impairs tetramer assembly. Using an in vitro translation system in the presence of mitochondria, Yokota et al. (38) observed that a K304R mutant was converted efficiently into tetramers, whereas a K304D mutant behaved like the K304E mutant. This also highlights the importance of the charge in the side chain of residue 304 . In our present work we addressed the question about this charge effect on stability and tetramer assembly by introduction of second site mutations replacing negatively charged residues in the vicinity of glutamic acid 304 in the folded structure. The second site mutant E300K/K304E did not rescue any of the effects of the K304E mutation, rather the effects of the mutations were additive. This indicates that disruption of the salt bridge between glutamic acid 300 and arginine 383 in the other subunit has strong effects on subunit assembly in itself and that the charge interaction between the side chains of residues 304 and 300 is less important.

Introduction of the $\mathrm{D} 346 \mathrm{~K}$ second site mutation reestablishes a high oligomer assembly efficiency. This is evident from the clearly higher amounts of active MCAD produced. The yield of active mutant enzyme was still clearly increased by improving the folding conditions, demonstrating that the effect on folding is not suppressed. The rescuing effect of the D346K mutation thus indicates that a charge repulsion effect between the carboxyl groups of glutamic acid 304 and aspartic acid 346 in the $\mathrm{K} 304 \mathrm{E}$ mutant is responsible for a distortion in the monomer structure which results in a surface unfavorable for subunit docking. The fact that the K304E mutant, with a lower but observable efficiency, does form oligomers suggests that the unfavorable alignment of the side chains can be overcome. This may require a rearrangement which demands a high energy intermediate state making it kinetically unfavorable. The K304Q mutant, with a constellation where there is neither charge attraction nor repulsion between residues 304 and 346 , also displays a relatively high oligomer assembly competence, further supporting the notion of a deleterious effect of the charge repulsion. The thermal stability results obtained for the D346K and K304E/D346K mutants indicate that aspartic acid 346 in itself is critical for the thermal stability.

The contribution of the observed decreased thermal stability of $\mathrm{K} 304 \mathrm{E}$ MCAD enzyme to the low level of K304E MCAD observed in patient cells is at present not clear. The temperature range at which the enzyme becomes inactivated is well above physiological temperatures. However, it reflects a higher plasticity of the mutant tetramers and is thus expected to result in a decreased half-life of the $\mathrm{K} 304 \mathrm{E} \mathrm{MCAD}$ enzyme. In the light of the fact that the protein degradation machineries in $E$. coli and eucaryotic mitochondria are homologous $(39,40)$, the finding that levels of up to $80 \%$ of the wild-type control could be obtained in $E$. coli for the K304E/D346K double mutant which displays a lower thermal stability than the K304E mutant, lead us to suggest that the effect of the K304E mutation on stability is of minor pathophysiological importance.

In an attempt to check the relevance of the results obtained in the bacterial expression system we analyzed the effect of cultivation at low temperature on the relative amounts of K304E mutant enzyme produced in lymphoblastoid cells from patients. Our results indicate that cultivation at low temperature has a small but measurable positive effect on the relative amount of the K304E mutant protein in these cells. The temperature effect can most likely be ascribed to an improvement of the folding conditions. An increase in the steady state level of 
functional protein at low cultivation temperature has also been observed for the prevalent $\Delta \mathrm{F} 508$ mutation in cystic fibrosis $(41,42)$.

The possibility of being able to modulate the yield of correctly folded and assembled mutant protein may thus provide clues to explain the variable phenotypical manifestation of MCAD deficiency. On the one hand, clinical symptoms usually occur only under conditions of fasting stress, and on the other hand, the frequency studies indicate that only a minority of the individuals homozygous for the K304E mutation are symptomatic (13). In most cases, attacks are precipitated by fasting stress accompanied by feverish infections (4). This means that the lipid metabolism is activated and synthesis of $\beta$-oxidation enzymes is up-regulated under conditions of elevated temperature. It can well be imagined that this poses high demands on the capacity of the folding machinery, and proteins with folding defects will be less competitive in this situation. Although this has to be substantiated further, it may be a common pattern in many inherited diseases. Such "conditional" mutations may be retained in the population as they do not abolish the function of the protein under normal conditions and only become detrimental under conditions of stress for the folding machinery.

\section{REFERENCES}

1. Beinert, H. (1963) Enzymes 7, 447-476

2. Izai, K., Uchida, Y., Orii, T., Yamamoto, S., and Hashimoto, T. (1992) J. Biol. Chem. 267, 1027-1033

3. Hale D. E and Bennett, M. (1992) J. Pediatr. 121, 1-11

4. Iafolla, A. K., Thompson, R. J., and Roe, C. R. (1994) J. Pediatr. 124, 409-415

5. Tanaka, K., Yokota, I., Coates, P. M., Strauss, A. W., Kelly, D. P., Zhang, Z. Gregersen, N., Andresen, B. S., Matsubara, Y., Curtis, D., and Chen, Y. T. (1992) Hum. Mutat. 1, 271-279

6. Andresen, B. S., Bross, P., Winter, V., Kølvraa, S., Jensen, T., Bolund, L. Duran, M., Kim, J.-J., Curtis, D., Divry, P., Vianey-Saban, C., and Gregersen, N. (1993) Am. J. Hum. Genet. 53, 730-739

7. Andresen, B. S., Jensen, T. G., Bross, P., Knudsen, I., Winter, V., Kølvraa, S., Bolund, L., Ding, J.-H., Chen, Y.-T., van Hove, J. L. K., Curtis, D., Yokota, I. Tanaka, K., Kim, J.J. P., and Gregersen, N.. (1994) Am. J. Hum. Genet. $54,975-988$

8. Gregersen, N., Andresen, B. S., Bross, P., Winter, V., Engst, S., Rüdiger, N. Christensen, E., Kelly, D., Strauss, A. W., Kolvraa, S., Bolund, L., and Ghisla, S. (1991) Hum. Genet. 86, 545-551

9. Matsubara, Y., Narisawa, K., Miyabayashi, S., Tada, K., Coates, P. M. Bachmann, C. Elsas, L. J., II, Pollitt, R. J., Rhead, W. J., and Roe, C. R. (1990) Biochem. Biophys. Res. Commun. 171, 498-505

10. Kelly, D. P., Whelan, A. J., Ogden, M. L., Alpers, R., Zhang, Z., Bellus, G., Gregersen, N., Dorland, L., and Strauss, A. W. (1990) Proc. Natl. Acad. Sci. U. S. A. 97, 9326-9240

11. Yokota, I., Indo, Y., Coates, P. M., and Tanaka, K. (1990) J. Clin. Invest. 86, $1000-1003$

12. Gregersen, N., Winter, V., Curtis, D., Deufel, T., Willems, P., Ponzone, A.
Ding, J. H., Yang, B. Z., Iafolla, A. K. Chen, Y. T., Roe, C. R., Kølvraa, S., Scheiderman, K., Andresen, B. S., Bross, P., and Bolund, L. (1993) Hum. Hered. 43, 342-350

13. Wilcken, B. Hammond J and Silink M (1994) Arch Dis. Child 70, 410-412 14. Hartl, F. U., Hlodan, R., and Langer, T. (1994) Trends. Biochem. Sci. 19, 20-25

15. Bross, P., Andresen, B. S., Winter, V., Kräutle, F., Jensen, T. G., Nandy, A. Kølvraa, S., Ghisla, S., Bolund, L., and Gregersen, N. (1993) Biochim. Biophys. Acta 1182, 264-274

16. Kim, J.-J. P., Wang, M., and Paschke R. (1993) Proc. Natl. Acad. Sci. U. S. A $90,7523-7527$

17. Saijo, T., Welch, W. J., and Tanaka, K. (1994) J. Biol. Chem. 269, 4401-4408

18. Duran, M., Mitchell, G., de Klerk, B. C. J, de Jager, J. P., Hofkamp, M. Bruinvis, L., Ketting, D., Saudubray, J. M., and Waldman, S. K. (1985) J. Pediatr. 107, 397-402

19. Jensen, T. G., Bross, P., Andresen, B. S., Lund, T, B., Kristensen, T. J., Jensen, U. B., Winter, V., Kølvraa, S., Gregersen, N, and Bolund, L. (1995) Hum. Mutat., in press

20. Mitraki, A., Danner, M., King, J., and Seckler, R. (1993) J. Biol. Chem. 268, 20071-20075

21. Gordon, C. L., and King, J. (1994) Genetics 136, 427-438

22. Gordon, C. L., Sather, S. K., Casjens, S., and King, J. (1994) J. Biol. Chem. 269, 27941-27951

23. Bross, P., Engst, S., Strauss, A. W., Kelly, D. P., Rasched, I., and Ghisla, S. (1990) J. Biol. Chem. 265, 7116-7119

24. Kuipers, O. P., Boot, H. J., and DeVos, W. M. (1991) Nucleic Acids Res. 19 4558

25. Goloubinoff, P., Gatenby, A. A., and Lorimer, G. H. (1989) Nature 337, 44-47 26. Lehman, T. C., Hale, D. E., Bhala, A., and Thorpe, C. (1990) Anal. Biochem. 186, 280-284

27. Bross, P., Jensen, T. G., Andresen, B. S., Kjeldsen, M., Nandy, A., Kølvraa, S. Ghisla, S., Bolund, L., and Gregersen, N. (1994) Biochem. Med. Metab. Biol. $52,36-44$

28. Kølvraa, S., Gregersen, N., Christensen, E., and Hobolth, N. (1982) Clin. Chim. Acta 126, 53-67

29. Kraulis, P. J. (1991) J. Appl. Cryst. 24, 946-950

30. Matsubara, Y., Indo, Y., Naito, E., Ozasa, H., Glassberg, R., Vockley, J., Ikeda Y., Kraus, J., and Tanaka, K. (1989) J. Biol. Chem. 264, 16321-16331

31. Indo, Y., Yang-Feng, T., Glassberg, R., and Tanaka, K. (1991) Genomics 11, $609-620$

32. Kelly, D. P., Kim, J.-J., Billadello, J. J., Hainline, B. E., Chu, T. W., and Strauss, A. W. (1987) Proc. Natl. Acad. Sci., U. S. A. 84, 4068-4072

33. Matsubara, Y., Kraus, J. P., Ozasa, H., Glassberg, R., Finocchiaro, G., Ikeda Y., Mole, J., Rosenberg, L. E., and Tanaka, K. (1987) J. Biol. Chem. 262 10104-10108

34. Naito, E., Ozasa, H., Ikeda, Y., and Tanaka, K. (1989) J. Clin. Invest. 83, 1605-1613

35. Matsubara, Y., Ito, M., Glassberg, R., Satyabhama, S., Ikeda, Y., and Tanaka K. (1990) J. Clin. Invest. 85, 1058-1064

36. Matthews, B. W. (1993) Curr. Opin. Struct. Biol. 3, 589-593

37. Naito, E., Indo, Y., and Tanaka, K. (1990) J. Clin. Invest. 85, 1575-1582

38. Yokota, I., Saijo, T., Vockley, J., and Tanaka, K. (1992) J. Biol. Chem. 267, 26004-26010

39. Goldberg, A. L. (1992) Eur. J. Biochem. 203, 9-23

40. Van Dyck, L., Pearce, D. A., and Sherman, F. (1994) J. Biol. Chem. 269, $238-242$

41. Denning, G. M., Anderson, M. P., Amara, J. F., Marshall, J., Smith, A. E., and Welsh, M. J., (1992) Nature 358, 761-764

42. Lukacs, G. L., Chang, X.-B., Bear, C., Kartner, N., Mohamed, A., Riordan, J. R., and Grinstein, S. (1993) J. Biol. Chem. 268, 21592-21598 